\title{
Cell therapy for diverse central nervous system disorders: inherited metabolic diseases and autism
}

\author{
Jessica M Sun ${ }^{1}$ and Joanne Kurtzberg ${ }^{1}$
}

The concept of utilizing human cells for the treatment of medical conditions is not new. In its simplest form, blood product transfusion as treatment of severe hemorrhage has been practiced since the 1800s. The advent of hematopoietic stem cell transplantation (HSCT) began with the development of bone marrow transplantation for hematological malignancies in the mid-1900s and is now the standard of care for many hematological disorders. In the past few decades, HSCT has expanded to additional sources of donor cells, a wider range of indications, and the development of novel cell products. This trajectory has sparked a rapidly growing interest in the pursuit of innovative cell therapies to treat presently incurable diseases, including neurological conditions. HSCT is currently an established therapy for certain neurologically devastating inherited metabolic diseases, in which engrafting donor cells provide lifelong enzyme replacement that prevents neurological deterioration and significantly extends the lives of affected children. Knowledge gained from the treatment of these rare conditions has led to refinement of the indications and timing of HSCT, the study of additional cellular products and techniques to address its limitations, and the investigation of cellular therapies without transplantation to treat more common neurological conditions, such as autism spectrum disorder.

G enetic and neurological conditions of childhood are incredibly challenging for all parties involved. Currently available therapies consistently fall short for affected children, leaving them with life-long disabilities and, often times, shortened lifespans. The persistent unmet need for novel therapeutic approaches for children with genetic and acquired neurological diseases and the rise of the field of regenerative medicine have sparked interest in the development of biological, cell-based therapies for these conditions. In this article, we will review the current status of cell therapies for two types of neurological conditions that manifest in childhood: hematopoietic stem cell transplantation (HSCT) in patients with inherited metabolic diseases (IMDs), and investigational cellular therapies in patients with autism spectrum disorder (ASD).

\section{INHERITED METABOLIC DISEASES}

IMDs are a heterogeneous group of genetic diseases. In most of these conditions, a genetic mutation results in lack of a particular enzyme, resulting in the accumulation of toxic substrates and/or disruption of normal cellular processes throughout the body. Many affected babies appear normal at birth but begin to develop neurological symptoms in infancy or early childhood. Though the time course varies, these diseases cause progressive neurological deterioration ultimately leading to death later in infancy or childhood.

Strategies to prevent disease progression in the IMDs have focused on replacing the missing enzyme either via recombinant enzyme replacement therapy (ERT) or HSCT. Intravenous ERT is available for selected lysosomal storage diseases and is effective in improving certain systemic disease manifestations. It has several limitations, however, including the need for lifelong intravenous infusions, the risk of an immune response to the recombinant enzyme that can render the infusion both dangerous and ineffective, and the inability to reach several tissues or organs in sufficient quantities. In particular, ERT cannot effectively cross the blood-brain barrier and therefore cannot prevent the progression of neurological symptoms $(1,2)$. Intrathecal ERT (3), chaperone technology (4-6), and gene therapy are under investigation as alternative methods to addre.ss this limitation.

Gene therapy for selected IMDs has been the subject of preclinical (7-11) and a few small human studies. Early-phase clinical trials of in vivo gene transfer, in which genetic material is delivered directly to a patient, are underway using adenovirus vectors in Hurler (NCT02702115), Hunter (NCT03041234), Sanfilippo (NCT02716246), and Maroteaux-Lamy (NCT03173521) syndromes, Batten disease (NCT01414985, NCT01161576, NCT00151216), and Metachromatic leukodystrophy (MLD, NCT01801709). In one study of 10 patients with Batten disease, intracranial injections of adenovirus-associated DNA failed to prevent disease progression, although the rate of neurological decline slowed somewhat. Four patients developed an immune response to the vector, and one patient developed status epilepticus 2 weeks after treatment and died a month later (12). Another gene therapy approach involves ex vivo manipulation of autologous or donor cells in vitro that are subsequently

\footnotetext{
${ }^{1}$ Robertson Clinical and Translational Cell Therapy Program, Duke University, Durham, North Carolina. Correspondence: Jessica M. Sun (jessica.sun@duke.edu) 
delivered to the patient. The only published data of this technique in IMDs involved autologous HSCT using CD34+ cells genetically modified via lentiviral transduction and then reinfused in patients with adrenoleukodystrophy (ALD) (13) or MLD $(14,15)$ after conditioning with busulfan+/-cyclophosphamide. The two patients with ALD showed an arrest of cerebral demyelination 12-16 months after treatment (13). The first nine patients with MLD treated with gene therapy demonstrated transduced cell engraftment of 14-95\% with improvement of enzyme levels in the peripheral blood and clinical benefit without evidence of antibody formation or malignancy with a median follow-up of 3 years (14). Followup of the entire study cohort is ongoing. Although gene therapy approaches have been encouraging in animal models, issues regarding the possibility of ongogenesis, development of immune responses to the vector or novel protein, and unclear ability of vectors and enzymes to traverse the bloodbrain barrier remain potential limitations in their translation to clinical use. Currently, the only effective therapy to halt neurological progression in certain IMDs is allogeneic HSCT.

\section{Mechanism of HSCT in IMDs}

Following successful HSCT, donor-derived cells engraft in the bone marrow and distribute throughout the body, including peripheral tissues and the central nervous system, and serve as a constant endogenous source of the missing enzyme, thereby slowing or halting the progression of disease $(16,17)$. The basis for this concept was first introduced in the 1960s by Elizabeth Neufeld, who demonstrated that co-culture of fibroblasts from patients with two different IMDs (Hunter and Hurler syndromes) resulted in correction of each condition via intercellular enzyme transport between cells (18). This phenomenon of 'cross-correction' is the primary mechanism of ERT and of cellular therapy used for that purpose. In the brain, donor-derived microglia cells of myeloid origin are thought to be the source of ERT after HSCT (19). These donor-derived cells secrete a portion of their lysosomal enzymes that can then be taken up by neighboring cells, thereby cross-correcting the metabolic defect in affected host cells (19-21). In addition to acting as a permanent source of ERT, engrafted donor-derived cells may potentially aid in reducing the burden of accumulated toxic substrates in the brain and/or exert anti-inflammatory and pro-neurogenic effects through paracrine signaling.

\section{Clinical Experience in Subtypes of IMDs}

There are hundreds of different IMDs. HSCT is currently indicated or under investigation for a subset of these disorders, including lysosomal storage diseases, peroxisomal storage diseases, and a few select others (see Table 1).

Table 1. IMDs for which HSCT is indicated or under investigation

\begin{tabular}{lcc}
\hline HSCT indicated* & HSCT investigational & ERT first-line therapy \\
\hline Lysosomal storage disorders & Lysosomal storage disorders & Lysosomal storage disorders \\
Mucoploysaccharidoses & Mucoploysaccharidoses & Mucoploysaccharidoses \\
Hurler (MPS IH), severe phenotype & Hunter (MPS II), with CNS disease & Hurler (MPS IH/S, IS), attenuated phenotypes \\
& Sanfilippo (MPS IIIA-D) & Hunter (MPS II), without CNS disease \\
& Morquio (MPS IV) & Maroteaux-Lamy (MPS VI) \\
Sphingolipidoses & Sly (MPS VII) & Sphingolipidoses \\
Globoid leukodystrophy (Krabbe) & Sphingolipidoses & Fabry \\
Metochromatic leukodystrophy (MLD) & GM1 gangliosidosis & Gaucher, types 1/3 \\
& GM2 Gangliosidosis, type 1 (Tay-Sachs) & \\
GM2 gangliosidosis, type 2 (Sandhoff) & Other \\
& Farber & Pompe
\end{tabular}

Oligosaccharidoses

Alpha-mannosidosis

Fucosidosis

Other LSDs

Mucolipidosis II (I-cell disease)

Wolman disease
Peroxisomal storage disorders

Adrenomyeloneuropathy

Other

Batten disease

Pelizaeus-Merzbacher disease

Peroxisomal storage disorders 
Mucopolysaccharidoses. The mucopolysaccharidoses (MPS) are lysosomal storage diseases in which a particular enzyme deficiency leads to progressive accumulation of incompletely degraded glycosaminoglycans in lysosomes, causing broad disease manifestations, including psychomotor retardation, musculoskeletal manifestations, vision and hearing impairment, and life-threatening cardiopulmonary failure. The first HSCT for an IMD was performed in 1980 in a 1-year-old child with Hurler syndrome (MPS1) using bone marrow from his parents (22). Since then, >500 transplants have been performed worldwide in patients with Hurler syndrome, making it the most transplanted and well-studied IMD.

Numerous reports have demonstrated the efficacy of both bone marrow and umbilical cord blood transplantation (CBT) in Hurler syndrome, including improvements in neurocognitive function, joint integrity, motor development, growth, hydrocephalus, corneal clouding, cardiac function, hepatosplenomegaly, hearing, visual and auditory processing, and overall survival (23-29). However, survivors still experience a variable degree of residual disease burden (30). Factors associated with superior clinical outcomes include transplantation early in the course of the disease and the ability to attain full donor chimerism and normal enzyme levels posttransplant $(30,31)$. HSCT in other MPS types has also been performed in small numbers and suggest benefit in Hunter and Sanfilippo syndromes $(32,33)$. Outcomes in other subtypes have been variable, raising the possibility that some MPS diseases may be more responsive to HSCT than others (34-41).

Leukodystrophies. Krabbe disease, a leukodystrophy caused by mutations in the enzyme GALC, leads to the accumulation of psychocine that causes apoptosis of myelin-forming oligodendrocytes and Schwann cells and increased inflammation in both the central and peripheral nervous systems. In the most common early infantile form, babies develop symptoms, including irritability, spasticity, developmental regression, and seizures within the first 6 months of life and die within 2 years. In 2005, the outcomes of 11 asymptomatic babies with Krabbe disease who underwent CBT in the first month of life were reported along with 14 infants transplanted after the onset of symptoms (42). With a median follow-up of 3 years, survival was dramatically increased in babies who were transplanted prior to the development of symptoms (100\% vs. $42.8 \%)$. Newborns who underwent transplant with minimal-to-no symptoms of disease exhibited substantial neurodevelopmental gains in all areas of development compared with symptomatic infants and untreated patients. Nonetheless, some degree of gross motor function deficit became apparent in all the children. A more recent analysis of late outcomes demonstrated that babies transplanted $<30$ days of age have superior outcomes than those transplanted at $>30$ days (43).

MLD is a lysosomal storage disease caused by a deficiency in the enzyme arylsulfatase A, leading to accumulation of sphingolipids and a progressive loss of myelinating cells. The time of onset and severity of symptoms correlate with residual arylsulfatase A activity and vary widely from early- or lateinfantile to juvenile and adult-onset forms. Neurological manifestations may include spasticity, neuropathy, dementia, seizures, and optic atrophy. Series of patients with MLD treated with HSCT report a 5-year overall survival of 59-74\% and suggest that later-onset phenotypes may benefit more, particularly if HSCT is performed early in the course of the disease $(44,45)$. Despite transplantation, may patients with MLD continue to experience progression of peripheral nervous system disease, although at a slower rate than expected by natural history.

$\mathrm{X}$-linked ALD is a peroxisomal disorder of very long-chain fatty acid metabolism, resulting in their accumulation in tissues and plasma. Symptoms include cerebral demyelination, adrenal insufficiency, and progressive neurological deterioration. Patients are followed for the development of symptoms clinically and radiographically. The Loes score, a severity scale used to determine the extent and damage to myelin in the brain via magnetic resonance imaging, is predictive of the course of disease progression and is used to aid treatment decisions (46). This score has also been associated with neurological outcome post-HSCT, with boys transplanted with a lower Loes score and less clinical symptoms faring better than those in later stages of the disease $(47,48)$.

\section{HSCT: Lessons Learned}

Although IMDs are rare, certain general principles have emerged from worldwide experience in HSCT for these conditions over the past few decades.

Limitations. Although HSCT has been effective in extending life for decades and preserving function in certain IMDs, it is currently ineffective in several IMDs and is not able to eradicate all sequelae of any given IMD. The degree of benefit varies among the different conditions and even among organ systems within a given condition. The reasons for this are still incompletely understood but include the possibility that thresholds of donor cell engraftment and enzyme production required to prevent disease progression differ between diseases and even between tissues in the same disease. Particular limitations are noted in diseases involving the peripheral nervous system, where peripheral nerve progression may occur despite stabilization of the central nervous system disease. Additional approaches are necessary to fully address the multifaceted tissue pathology in these diseases and normalize functional outcomes for patients. Augmented cellular therapies, such as CB-derived microglial-like cells (DUOC-01) (49-51) and others (52), gene therapies (15), supplemental enzyme therapy (53), and chaperone therapy, alone or in combination with HSCT, are all being investigated for this purpose.

Considerations of graft source and conditioning regimens. Particularly in Hurler syndrome, the IMD with the most data available, improved outcomes have been 
associated with the ability to attain full donor chimerism and normal enzyme levels posttransplant $(30,31)$. As most IMDs are inherited in an autosomal-recessive manner, many relatives are heterozygous carriers and therefore have lower than normal levels of the affected enzyme, making them less favorable donors. A retrospective study conducted by the European Group for Blood and Marrow Transplantation demonstrated statistically significant increases in both full-donor chimerism ( $93 \%$ vs. $67 \%$ ) and normal enzyme levels ( $100 \%$ vs. $72 \%$ ) when umbilical CB was utilized as a graft source compared with bone marrow or peripheral blood stem cells (54), and these high incidence levels have been reported in other series of CBT in Hurler syndrome and other lysosmal storage diseases $(23,32,34,42,55)$. Among patients receiving CBT for Hurler syndrome, a shorter interval between diagnosis and CBT ( $<4.6$ months $82 \%$ vs. $>4.6$ months $57 \%$ ) and a conditioning regimen containing busulfan and cyclophosphamide (busulfan/ cyclophosphamide $75 \%$ vs. other $44 \%$ ) have been associated with a significantly higher event-free survival (56).

Based on these observations, current guidelines developed by the European Group for Blood and Marrow Transplantation for HSCT in MPS patients prioritize CB as a donor source in the absence of a non-carrier matched sibling or fully matched unrelated donor and recommend myeloablative conditioning with busulfan/cyclophosphamide (later replaced with busulfan/fludarabine) with exposure-targeted intravenous bulsufan. Since these guidelines were instituted in 2005, transplant outcomes in patients with Hurler syndrome have improved significantly. Engrafted survival rates are now 95\%, with low transplant-related toxicity (29). As a result, fully matched CB grafts are now considered one of the most appealing cell sources, if not the most appealing cell source, for HSCT in patients with MPS.

Timing. Time is critical in the treatment of IMDs. The most favorable outcomes are achieved when patients undergo HSCT early in the course of the disease, either before symptoms develop or when they have minimal evidence of neurological disease $(27,42,45)$. This has multiple implications. It is imperative to establish an accurate diagnosis as soon as possible. To that end, newborn screening for Krabbe disease has been implemented in six states. In addition to identifying eight patients with early infantile Krabbe disease, approximately 100 novel mutations of unknown significance have been identified in the GALC gene. Thus, while newborn screening may lead to diagnosis in the presymptomatic state, thereby enabling transplantation earlier in life, it has also highlighted the challenges of implementing a diagnostically challenging screening program and counseling families and providers regarding indeterminate results.

After a diagnosis has been made and a through evaluation completed, minimizing the time to transplant is often critical. In addition to advantages of $\mathrm{CB}$ as a donor source in terms of post-HSCT chimerism and enzyme levels, $\mathrm{CB}$ is readily available and has less stringent HLA matching criteria, often making it the most time efficient and thus preferable donor source.
Even after HSCT, the timing of migration and engraftment of donor-derived cells in the brain is unknown. Based on clinical observations, however, it is likely several months after hematological engraftment. As a result, patients often experience a progressive loss of neurological function for the first few months after HSCT before sufficient numbers of donor cells engraft in the brain and produce adequate levels of the deficient enzyme resulting in disease stabilization. Most patients are left with some degree of residual and irreversible neurological impairment. To try to address that issue, a CBderived microglial-like cell product (DUOC-01) is under investigation at Duke University. This product is being tested in a phase I study as an intrathecal injection 4-6 weeks after CB transplant (NCT02254863).

Collaboration. Given the rarity and complexity of the IMDs, progress has only been made possible through the worldwide collaboration of multiple centers and disciplines. International registries, guidelines, and cooperative studies will be key to further refining indications for HSCT, optimizing transplant procedures, and developing approaches to address the current limitations of HSCT.

\section{AUTISM SPECTRUM DISORDER}

ASD, a neurodevelopmental disorder with onset in early childhood, is characterized by repetitive behaviors, a restricted range of activities, and impairments in social communication (57). It is a common disorder with a male predominance, with $>2$ million Americans and approximately 1 in 68 American children identified as falling on the autism spectrum (58). ASD is often accompanied by intellectual disability and is typically a chronic, disabling disorder. Though the severity of symptoms varies, the majority of individuals with ASD are unable to live independently and require lifelong support or accommodations, resulting in a societal cost of \$1.4-\$2.4 million per person (59). Current treatments of ASD are often multimodal, including countless hours of behavior, occupational, and speech therapies as well as specialized educational and vocational assistance and certain medications. These approaches target specific symptoms, such as irritability, that are associated with ASD, but they do not modify the underlying disease. Thus there is a substantial need for novel, effective, disease-modifying medical treatments for ASD.

The etiology of ASD remains unknown, though evidence suggests that it is likely to result from a complex interplay between genetic and environmental risk factors, potentially mediated through inflammatory and/or immune processes. Both animal models and observational human studies have linked immune activation in pregnant mothers to the development of ASD in their offspring. In addition, many cytokines and molecules classically associated with immune regulation are now also recognized as having roles in normal neurodevelopment. Thus, one hypothesis regarding the development of ASD is that immune-mediated changes in fetal brain cytokine profiles may result in abnormal development in the central nervous system, either directly or 


\section{Review | Sun and Kurtzberg}

indirectly via microglial activation. Abnormalities in the number, function, and gene regulation of microglia as well as in localized brain inflammation, pathological astrocyte activation, and synaptic dysfunction have all been described in various models of ASD (60-62).

\section{Potential Mechanisms of Action}

Cellular therapies are currently under investigation in clinical and preclinical studies with the goal of improving the core symptoms of ASD. There are multiple paracrine mechanisms through which cell therapies could potentially exert therapeutic effects. Cell-mediated immunomodulation, possibly via inhibition of $\mathrm{T}$-cell proliferation and reduced production of pro-inflammatory cytokines such as tumor necrosis factor$\alpha$ and interferon gamma (63), may reduce ongoing inflammation. Additional neuroprotection may be offered via molecular mechanisms by inhibiting toxic processes, such as neural apoptosis, microglial activation, astrocyte proliferation, and production of oxidative stress molecules (64). Cells may also have a role in stimulating the restoration and/or generation of functional synaptic pathways (65). These and other potential mechanisms may not be mutually exclusive and are the subject of ongoing research. Additional knowledge regarding the pathophysiology of ASD will be essential in further defining mechanisms by which cellular therapies might provide beneficial effects. This will also allow for further refinement of cell type, timing, and duration of therapy that may be useful.

\section{Animal Models}

As a disorder primarily of human social behavior and communication, establishing robust animal models of ASD is challenging. Many preclinical models have been developed utilizing mouse or rat models with single gene disorders associated with ASD, such as Fragile X, Rett, and Angelman syndromes. Studies of cell therapy approaches have been conducted in these models, though the results may not be generalizable to individuals with idiopathic ASD.

Rett syndrome is an X-linked condition associated with ASD that is typically caused by a mutation of the MECP2 gene, which encodes a methyl-CpG-binding protein. Mutations in $M E C P 2$ lead to deficient phagocytic function in glial cells. In a mouse model of Rett syndrome (Mecp2-null C57BL/6 mice), intravenous infusion of wild-type bone marrow cells arrested disease development. Following engraftment, survival was improved, breathing patterns normalized, apneas were reduced, body weight increased, and locomotor activity was improved, indicating functional recovery (66).

Another mouse model of ASD is the BTBR $T^{+} \operatorname{Itpr} 3^{t f} I$ (BTBR) mouse strain, derived from the inbred Black and Tan BRachyury strain. In addition to impaired social behavior, aberrant communication, increased repetitive behaviors, and increased cognitive rigidity, BTBR mice also exhibit increased levels of peripheral CD4+ T cells, peripheral B cells, and serum and brain immunoglobulin levels, among other immune abnormalities (67). Following intraventricular injection of human mesenchymal stromal cells into the central nervous system of BTBR mice undergoing concomitant immunosuppression with cyclosporine, improvements in all three ASD domains-social behavior, stereotyped behaviors, and cognitive rigidity-were observed in MSC-treated mice compared with controls. Differences in anxiety-related behaviors and locomotion were not observed (68).

These mouse models suggest that, at least in certain subtypes of ASD, cellular therapies may have the potential for benefit. Although chemical models utilizing administration of valproic acid during crucial periods of neurodevelopment and environmental models of maternal infection and inflammation exist in animals, there are no reports of the effect of cellular treatment in these models.

\section{Clinical Trials}

Clinical trials of cell therapy in patients with ASD are still in the early phases, with a handful of exploratory studies underway in several different countries. Cell sources for these studies include autologous (69) or allogeneic umbilical CB, autologous bone marrow (70), fetal stem cells (71), and mesenchymal stromal cells derived from adipose tissue or umbilical cord tissue. To date, results of four clinical trials have been published.

In India, 32 patients aged 3-33 years were treated with an intrathecal injection of autologous bone marrow mononuclear cells with a mean cell dose of $8.19 \times 10^{7}$ (70). Procedurerelated adverse events included headache (3.6\%), nausea (10.7\%), vomiting (17.9\%), pain (7.1\%), and aspiration (7.1\%). In addition, new or worsening seizures were observed in $9 \%$ of patients, as well as increased hyperactivity in $18.7 \%$. These side effects were likely due to the intrathecal route of administration. Statistically significant improvements were detected in the Clinical Global Impression-Improvement scale and Indian Scale for Assessment of Autism at the time of follow-up, which ranged from 5 to 26 months.

In Ukraine, the safety of intravenous and subcutaneous infusions of fetal stem cells was tested in a total of 45 children, aged 3-15 years (71). Patients were followed at 6 and 12 months after treatment, with improvement noted in speech, sociability, sensory, and overall health domains of the Autism Treatment Evaluation Checklist and the Aberrant Behavior Checklist (ABC). No adverse events were observed during the 1-year follow-up period, which may not be a sufficient duration of follow-up for fetal stem cells.

In the United States, the Duke ABCs trial was an open-label phase 1 safety and tolerability study of a single intravenous infusion of autologous CB conducted in 25 children, aged 2-5 years, with ASD (ClinicalTrials.gov ID: NCT02176317) (69). $\mathrm{CB}$ was administered as a single infusion (median infused dose: $2.6 \times 10^{7} / \mathrm{kg}$, range: $1.0 \times 10^{7} / \mathrm{kg}-8.1 \times 10^{7} / \mathrm{kg}$ ) without any immunosuppression. The infusions were safe, with no serious adverse events and occasional allergic reactions and irritability reported. Improvements in ASD symptoms were observed on caregiver-completed measures (Vineland 


\section{Inherited metabolic diseases and autism $\mid$ ReVieW}

a

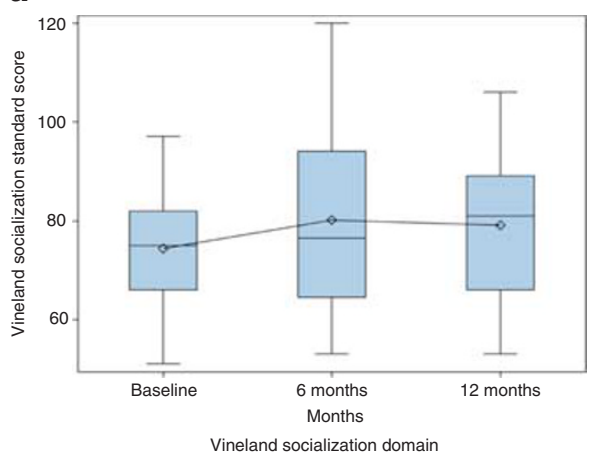

b

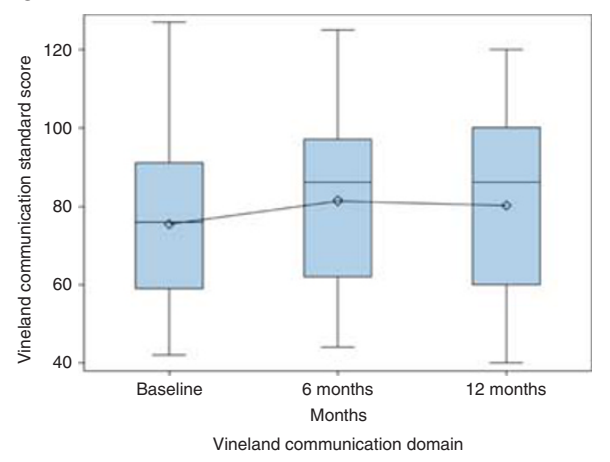

c

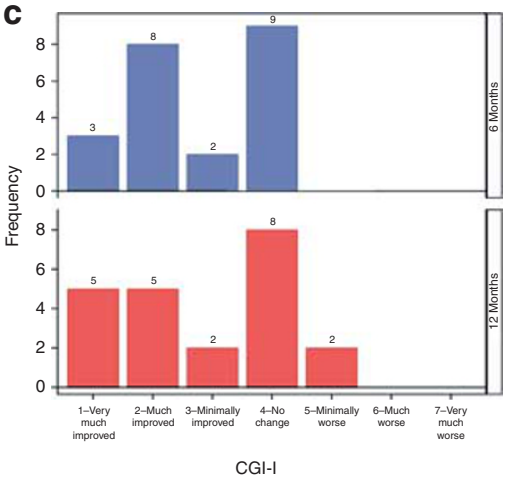

Figure 1. Changes in autism spectrum disorder symptoms after autologous cord blood infusion. (a) Vineland-Il socialization domain standard scores at baseline and 6 months after infusion ( $P=0.02$ baseline to 6 months). (b) Vineland-II communication domain standard scores at baseline and 6 months after infusion $(P<0.01$ baseline to 6 months). (c) Distribution of Clinical Global Impression-Improvement scale (CGI-I) scores at 6 (blue) and 12 (red) months postinfusion. Sample sizes are $N=25$ for baseline and 6-month time points and $N=22$ at 12 months.

Adaptive Behavior Scales-Second Edition (see Figure 1) and Pervasive Developmental Disorder Behavior Inventory), clinician assessment (Clinical Global ImpressionImprovement scale, Figure 1), and computerized eyetracking assessments. Positive changes, including increased social communication skills and receptive/expressive language and decreased repetitive behavior and sensory sensitivities were observed 6 months after infusion and maintained at 12 months. A phase 2 randomized study is underway to evaluate the efficacy of autologous or allogeneic CB therapy vs. placebo in children with ASD (NCT02847182).

Owing to their well-established immunomodulatory capacity, mesenchymal stromal cells may be an excellent candidate cell type for use in the treatment of ASD. One Chinese study has evaluated the administration of MSCs in children with ASD in conjunction with additional cells. In that trial, 37 children with ASD were treated with 4 doses of either umbilical cord blood mononuculear cells (given both intravenously and intrathecally) $(n=14)$, cord blood mononuculear cells+intrathecal umbilical cord-derived mesenchymal stem cells $(n=9)$, or standard therapy $(n=14)(72)$. The only treatment-related side effect was transient fever in five patients. At 6 months posttreatment, both treated groups demonstrated greater improvement in multiple ASD measures (Childhood Autism Rating Scale, Clinical Global Impression scale, $\mathrm{ABC}$ ) than the placebo group, indicating a potential therapeutic response. A phase I study of intravenous administration of umbilical cord-derived MSCs in children with ASD is underway at Duke University in the United States.

\section{SUMMARY}

Although adequate treatments for many neurological disorders of childhood remain elusive, cellular therapies have potential as novel therapeutic modalities to transform the way we approach these conditions. Significant advances in the filed of HSCT for IMDs have resulted in improved survival rates and neurological outcomes in conditions that previously were universally fatal. This serves as an example of the advancements that can be made through scientific discovery, innovation, and collaboration. Although cell therapy has yet to be established as a treatment for other neurological conditions such as cerebral palsy or ASD, there is mounting preclinical and clinical evidence of potential benefit and important advances are anticipated in the coming years. Should cell therapy prove beneficial in these acquired conditions via immune modulation, neuroprotection, or other mechanisms, then there may be applications in more common conditions including, but not limited to, adult neurodegenerative diseases, multiple sclerosis, and stroke.

Disclosure: The authors declare no conflict of interest.

\section{REFERENCES}

1. Shull RM, Kakkis ED, McEntee MF, Kania SA, Jonas AJ, Neufeld EF. Enzyme replacement in a canine model of Hurler syndrome. Proc Natl Acad Sci USA 1994;91:12937-41.

2. Tokic V, Barisic I, Huzjak N, Petkovic G, Fumic K, Paschke E. Enzyme replacement therapy in two patients with an advanced severe (Hurler) phenotype of mucopolysaccharidosis I. Eur J Pediatr 2007;166:727-32.

3. Dickson PI, Kaitila I, Harmatz P, et al. Safety of laronidase delivered into the spinal canal for treatment of cervical stenosis in mucopolysaccharidosis I. Mol Genet Metab 2015;116:69-74.

4. Valenzano KJ, Khanna R, Powe AC, et al. Identification and characterization of pharmacological chaperones to correct enzyme deficiencies in lysosomal storage disorders. Assay Drug Dev Technol 2011;9:213-35.

5. Warnock DG, Bichet DG, Holida M, et al. Oral migalastat $\mathrm{HCl}$ leads to greater systemic exposure and tissue levels of active alpha-galactosidase A in Fabry patients when co-administered with infused agalsidase. PLoS ONE 2015;10:e0134341.

6. Xu S, Lun Y, Brignol N, et al. Coformulation of a novel human alphagalactosidase a with the pharmacological chaperone AT1001 leads to improved substrate reduction in Fabry mice. Mol Ther 2015;23:1169-81.

7. Gentner B, Visigalli I, Hiramatsu H, et al. Identification of hematopoietic stem cell-specific miRNAs enables gene therapy of globoid cell leukodystrophy. Sci Transl Med 2010;2:58ra84.

8. Meneghini V, Lattanzi A, Tiradani L, et al. Pervasive supply of therapeutic lysosomal enzymes in the CNS of normal and Krabbeaffected non-human primates by intracerebral lentiviral gene therapy. EMBO Mol Med 2016;8:489-510. 
9. Rosenberg JB, Kaminsky SM, Aubourg P, Crystal RG, Sondhi D. Gene therapy for metachromatic leukodystrophy. J Neurosci Res 2016;94: 1169-79.

10. Ruiz de Garibay AP, Solinis MA, Rodriguez-Gascon A. Gene therapy for fabry disease: a review of the literature. Biodrugs 2013;27:237-46.

11. Zerah M, Piguet F, Colle MA, et al. Intracerebral gene therapy using AAVrh.10-hARSA recombinant vector to treat patients with early-onset forms of metachromatic leukodystrophy: preclinical feasibility and safety assessments in nonhuman primates. Hum Gene Ther Clin Dev 2015;26: $113-24$.

12. Worgall S, Sondhi D, Hackett NR, et al. Treatment of late infantile neuronal ceroid lipofuscinosis by CNS administration of a serotype 2 adeno-associated virus expressing CLN2 cDNA. Hum Gene Ther 2008;19:463-74.

13. Cartier N, Hacein-Bey-Abina S, Bartholomae CC, et al. Lentiviral hematopoietic cell gene therapy for X-linked adrenoleukodystrophy. Methods Enzymol 2012;507:187-98.

14. Sessa M, Lorioli L, Fumagalli F, et al. Lentiviral haemopoietic stem-cell gene therapy in early-onset metachromatic leukodystrophy: an ad-hoc analysis of a non-randomised, open-label, phase $1 / 2$ trial. Lancet 2016;388:476-87.

15. Biffi A, Montini E, Lorioli L, et al. Lentiviral hematopoietic stem cell gene therapy benefits metachromatic leukodystrophy. Science 2013;341: 1233158.

16. Di Ferrante N, Nichols BL, Donnelly PV, Neri G, Hrgovcic R, Berglund RK. Induced degradation of glycosaminoglycans in Hurler's and Hunter's syndromes by plasma infusion. Proc Natl Acad Sci USA 1971;68:303-7.

17. Knudson AG Jr., Di Ferrante N, Curtis JE. Effect of leukocyte transfusion in a child with type II mucopolysaccharidosis. Proc Natl Acad Sci USA 1971;68:1738-41.

18. Fratantoni JC, Hall CW, Neufeld EF. Hurler and Hunter syndromes: mutual correction of the defect in cultured fibroblasts. Science 1968;162: $570-2$.

19. Krivit W, Sung JH, Shapiro EG, Lockman LA. Microglia: the effector cell for reconstitution of the central nervous system following bone marrow transplantation for lysosomal and peroxisomal storage diseases. Cell Transplant 1995;4:385-92.

20. Unger ER, Sung JH, Manivel JC, Chenggis ML, Blazar BR, Krivit W. Male donor-derived cells in the brains of female sex-mismatched bone marrow transplant recipients: a Y-chromosome specific in situ hybridization study. J Neuropathol Exp Neurol 1993;52:460-70.

21. Neufeld EF, MJ. The mucopolysaccharidoses. In: Scriver CR BA, Sly W, Valle D eds. The Metabolic and Molecular Bases of Inherited Disease, 8th edn. McGraw-Hill, 2001: 3421-52.

22. Hobbs JR, Hugh-Jones K, Barrett AJ, et al. Reversal of clinical features of Hurler's disease and biochemical improvement after treatment by bonemarrow transplantation. Lancet 1981;2:709-12.

23. Staba SL, Escolar ML, Poe M, et al. Cord-blood transplants from unrelated donors in patients with Hurler's syndrome. N Engl J Med 2004;350:1960-9.

24. Peters C, Shapiro EG, Anderson J, et al. Hurler syndrome: II. Outcome of HLA-genotypically identical sibling and HLA-haploidentical related donor bone marrow transplantation in fifty-four children. The Storage Disease Collaborative Study Group. Blood 1998;91:2601-8.

25. Souillet G, Guffon N, Maire I, et al. Outcome of 27 patients with Hurler's syndrome transplanted from either related or unrelated haematopoietic stem cell sources. Bone Marrow Transplant 2003;31:1105-17.

26. Bjoraker KJ, Delaney K, Peters C, Krivit W, Shapiro EG. Long-term outcomes of adaptive functions for children with mucopolysaccharidosis I (Hurler syndrome) treated with hematopoietic stem cell transplantation. J Dev Behav Pediatr 2006;27:290-6.

27. Boelens JJ, Wynn RF, O'Meara A, et al. Outcomes of hematopoietic stem cell transplantation for Hurler's syndrome in Europe: a risk factor analysis for graft failure. Bone Marrow Transplant 2007;40:225-33.
28. Aldenhoven M, Boelens JJ, de Koning TJ. The clinical outcome of Hurler syndrome after stem cell transplantation. Biol Blood Marrow Transplant 2008;14:485-98.

29. Aldenhoven M, Jones SA, Bonney D, et al. Hematopoietic cell transplantation for mucopolysaccharidosis patients is safe and effective: results after implementation of international guidelines. Biol Blood Marrow Transplant 2015;21:1106-9.

30. Aldenhoven M, Wynn RF, Orchard PJ, et al. Long-term outcome of Hurler syndrome patients after hematopoietic cell transplantation: an international multicenter study. Blood 2015;125:2164-72.

31. Boelens JJ, Aldenhoven M, Purtill D, et al. Outcomes of transplantation using various hematopoietic cell sources in children with Hurler syndrome after myeloablative conditioning. Blood 2013;121:3981-7.

32. Prasad VK, Mendizabal A, Parikh SH, et al. Unrelated donor umbilical cord blood transplantation for inherited metabolic disorders in 159 pediatric patients from a single center: influence of cellular composition of the graft on transplantation outcomes. Blood 2008;112:2979-89.

33. Tanaka A, Okuyama T, Suzuki Y, et al. Long-term efficacy of hematopoietic stem cell transplantation on brain involvement in patients with mucopolysaccharidosis type II: a nationwide survey in Japan. Mol Genet Metab 2012;107:513-20.

34. Martin PL, Carter SL, Kernan NA, et al. Results of the cord blood transplantation study (COBLT): outcomes of unrelated donor umbilical cord blood transplantation in pediatric patients with lysosomal and peroxisomal storage diseases. Biol Blood Marrow Transplant 2006;12: 184-94.

35. Boelens JJ. Trends in haematopoietic cell transplantation for inborn errors of metabolism. J Inherit Metab Dis 2006;29:413-20.

36. Annibali R, Caponi L, Morganti A, Manna M, Gabrielli O, Ficcadenti A. Hunter syndrome (Mucopolysaccharidosis type II), severe phenotype: long term follow-up on patients undergone to hematopoietic stem cell transplantation. Minerva Pediatr 2013;65:487-96.

37. Guffon N, Bertrand Y, Forest I, Fouilhoux A, Froissart R. Bone marrow transplantation in children with Hunter syndrome: outcome after 7 to 17 years. J Pediatr 2009;154:733-7.

38. Jester S, Larsson J, Eklund EA, et al. Haploidentical stem cell transplantation in two children with mucopolysaccharidosis VI: clinical and biochemical outcome. Orphanet J Rare Dis 2013;8:134.

39. Peters C, Steward CG, National Marrow Donor Program, International Bone Marrow Transplant Registry, Working Party on Inborn Errors European Bone Marrow Transplant Group. Hematopoietic cell transplantation for inherited metabolic diseases: an overview of outcomes and practice guidelines. Bone Marrow Transplant 2003;31:229-39.

40. Vellodi A, Young E, Cooper A, Lidchi V, Winchester B, Wraith JE. Longterm follow-up following bone marrow transplantation for Hunter disease. J Inherit Metab Dis 1999;22:638-48.

41. Vellodi A, Young E, New M, Pot-Mees C, Hugh-Jones K. Bone marrow transplantation for Sanfilippo disease type B. J Inherit Metab Dis 1992;15: 911-8.

42. Escolar ML, Poe MD, Provenzale JM, et al. Transplantation of umbilicalcord blood in babies with infantile Krabbe's disease. N Engl J Med 2005;352:2069-81.

43. Allewelt HB, Page $\mathrm{K}$, Taskindoust $\mathrm{M}$, et al. Long-term functional outcomes following hematopoietic stem cell transplantation for Krabbe disease. Biol Blood Marrow Transplant 22:S102-3.

44. Boucher AA, Miller W, Shanley R, et al. Long-term outcomes after allogeneic hematopoietic stem cell transplantation for metachromatic leukodystrophy: the largest single-institution cohort report. Orphanet J Rare Dis 2015;10:94.

45. Martin HR, Poe MD, Provenzale JM, Kurtzberg J, Mendizabal A, Escolar ML. Neurodevelopmental outcomes of umbilical cord blood transplantation in metachromatic leukodystrophy. Biol Blood Marrow Transplant 2013;19:616-24.

46. Loes DJ, Fatemi A, Melhem ER, et al. Analysis of MRI patterns aids prediction of progression in X-linked adrenoleukodystrophy. Neurology 2003;61:369-74. 
47. Miller WP, Rothman SM, Nascene D, et al. Outcomes after allogeneic hematopoietic cell transplantation for childhood cerebral adrenoleukodystrophy: the largest single-institution cohort report. Blood 2011;118:1971-8.

48. Beam D, Poe MD, Provenzale JM, et al. Outcomes of unrelated umbilical cord blood transplantation for X-linked adrenoleukodystrophy. Biol Blood Marrow Transplant 2007;13:665-74.

49. Tracy E, Aldrink J, Panosian J, et al. Isolation of oligodendrocyte-like cells from human umbilical cord blood. Cytotherapy 2008;10:518-25.

50. Tracy ET, Zhang CY, Gentry T, Shoulars KW, Kurtzberg J. Isolation and expansion of oligodendrocyte progenitor cells from cryopreserved human umbilical cord blood. Cytotherapy 2011;13:722-9.

51. Kurtzberg J, Buntz S, Gentry T, et al. Preclinical characterization of DUOC-01, a cell therapy product derived from banked umbilical cord blood for use as an adjuvant to umbilical cord blood transplantation for treatment of inherited metabolic diseases. Cytotherapy 2015;17:803-15.

52. Koc ON, Day J, Nieder M, Gerson SL, Lazarus HM, Krivit W. Allogeneic mesenchymal stem cell infusion for treatment of metachromatic leukodystrophy (MLD) and Hurler syndrome (MPS-IH). Bone Marrow Transplant 2002;30:215-22.

53. Li Y, Sands MS. Experimental therapies in the murine model of globoid cell leukodystrophy. Pediatr Neurol 2014;51:600-6.

54. Boelens JJ, Wynn RF, O'Meara A, et al. Outcomes of hematopoietic stem cell transplantation for Hurler's syndrome in Europe: a risk factor analysis for graft failure. Bone Marrow Transplant 2007;40:225-33.

55. Church H, Tylee K, Cooper A, et al. Biochemical monitoring after haemopoietic stem cell transplant for Hurler syndrome (MPSIH): implications for functional outcome after transplant in metabolic disease. Bone Marrow Transplant 2007;39:207-10.

56. Boelens JJ, Rocha V, Aldenhoven M, et al. Risk factor analysis of outcomes after unrelated cord blood transplantation in patients with hurler syndrome. Biol Blood Marrow Transplant 2009;15:618-25.

57. King BH, Navot N, Bernier R, Webb SJ. Update on diagnostic classification in autism. Curr Opin Psychiatry 2014;27:105-9.

58. Developmental Disabilities Monitoring Network Surveillance Year 2010 Principal InvestigatorsCenters for Disease Control and PreventionPrevalence of autism spectrum disorder among children aged 8 years - autism and developmental disabilities monitoring network, 11 sites, United States, 2010MMWR Surveill Summ 2014;63:1-21.
59. Buescher AV, Cidav Z, Knapp M, Mandell DS. Costs of autism spectrum disorders in the United Kingdom and the United States. JAMA Pediatr 2014;168:721-8.

60. Takano T. Role of microglia in autism: recent advances. Dev Neurosci 2015;37:195-202.

61. Zantomio D, Chana G, Laskaris L, et al. Convergent evidence for mGluR5 in synaptic and neuroinflammatory pathways implicated in ASD. Neurosci Biobehav Rev 2015;52:172-7.

62. Goines PE, Ashwood P Cytokine dysregulation in autism spectrum disorders (ASD): possible role of the environment. Neurotoxicol Teratol 2013;36:67-81.

63. Vellasamy S, Tong CK, Azhar NA, et al. Human mesenchymal stromal cells modulate $\mathrm{T}$-cell immune response via transcriptomic regulation. Cytotherapy 2016;18:1270-83.

64. Gesundheit B, Ashwood P, Keating A, Naor D, Melamed M, Rosenzweig JP. Therapeutic properties of mesenchymal stem cells for autism spectrum disorders. Med Hypotheses 2015;84:169-77.

65. Koh S, Kim N, Yin HH, Harris IR, Dejneka NS, Eroglu C. Human umbilical tissue-derived cells promote synapse formation and neurite outgrowth via thrombospondin family proteins. J Neurosci 2015;35:15649-65.

66. Derecki NC, Cronk JC, Lu Z, et al. Wild-type microglia arrest pathology in a mouse model of Rett syndrome. Nature 2012;484:105-9.

67. Heo Y, Zhang Y, Gao D, Miller VM, Lawrence DA. Aberrant immune responses in a mouse with behavioral disorders. PLoS ONE 2011;6: e20912.

68. Segal-Gavish H, Karvat G, Barak N, et al. Mesenchymal stem cell transplantation promotes neurogenesis and ameliorates autism related behaviors in BTBR mice. Autism Res 2016;9:17-32.

69. Dawson G, Sun JM, Davlantis KS, et al. Autologous cord blood infusions are safe and feasible in young children with autism spectrum disorder: results of a single-center phase I open-label trial. Stem Cells Transl Med 2017;6:1332-9.

70. Sharma A, Gokulchandran N, Sane H, et al. Autologous bone marrow mononuclear cell therapy for autism: an open label proof of concept study. Stem Cells Int 2013;2013:623875.

71. Bradstreet JJ, Sych N, Antonucci N, et al. Efficacy of fetal stem cell transplantation in autism spectrum disorders: an open-labeled pilot study. Cell Transplant 2014;23 (Suppl 1): S105-12.

72. Lv YT, Zhang Y, Liu M, et al. Transplantation of human cord blood mononuclear cells and umbilical cord-derived mesenchymal stem cells in autism. J Transl Med 2013;11:196. 